\title{
PMI: The Photospheric Magnetic Field Imager
}

\author{
Jan Staub ${ }^{1, *}$, German Fernandez-Rico ${ }^{1}$, Achim Gandorfer ${ }^{1}$, Laurent Gizon ${ }^{1,4,5}$, \\ Johann Hirzberger ${ }^{1}$, Stefan $\mathrm{Kraft}^{3}$, Andreas Lagg ${ }^{1}$, Jesper Schou ${ }^{1}$, Sami K. Solanki ${ }^{1,6}$, \\ Jose Carlos del Toro Iniesta ${ }^{2}$, Thomas Wiegelmann ${ }^{1}$, and Joachim Woch ${ }^{1}$ \\ ${ }^{1}$ Max-Planck-Institut für Sonnensystemforschung, Justus-von-Liebig-Weg 3, 37077 Göttingen, Germany \\ 2 Instituto de Astrofísica de Andalucía (IAA-CSIC), Apartado de Correos 3004, 18080 Granada, Spain \\ ${ }^{3}$ ESOC - ESA, Robert-Bosch-Strasse 5, 64293 Darmstadt, Germany \\ ${ }^{4}$ Georg-August-Universität Göttingen, Institut für Astrophysik, Friedrich-Hund-Platz 1, 37077 Göttingen, Germany \\ ${ }^{5}$ Center for Space Science, NYUAD Institute, New York University Abu Dhabi, PO Box 129188, Abu Dhabi, UAE \\ ${ }^{6}$ School of Space Research, Kyung Hee University, Yongin, 446-701 Gyeonggi-Do, Korea
}

Received 16 June 2020 / Accepted 9 October 2020

\begin{abstract}
We describe the design and the capabilities of the Photospheric Magnetic field Imager (PMI), a compact and lightweight vector magnetograph, which is being developed for ESA's Lagrange mission to the Lagrange L5 point. After listing the design requirements and give a scientific justification for them, we describe the technical implementation and the design solution capable of fulfilling these requirements. This is followed by a description of the hardware architecture as well as the operations principle. An outlook on the expected performance concludes the paper.
\end{abstract}

Keywords: Sun: magnetic fields / Sun: activity / techniques: polarimetric / instrumentation: magnetographs

\section{Introduction}

Space weather monitoring, and especially space weather forecasts rely on the modeling of the magnetic field structure in the outer layers of the solar atmosphere. The main pillar of such modeling is extrapolation of the magnetic field using the photospheric magnetic field as the lower boundary condition. Only here can the magnetic field be quantitatively measured with sufficient accuracy for this task, using Zeeman magnetography, a technique requiring spectro-polarimetric measurements. Although significant technical progress must be acknowledged in our attempts to measure the magnetic field vector also in chromospheric layers (for a recent review see c.f. Lagg et al., 2017 and references therein), it is fair to say that a reliable and quantitative analysis of the magnetic field vector is still restricted to the photosphere, at least for full-disk measurements.

So far, all of our modelling efforts suffer to a large extent from two main limitations. Firstly, in the majority of extrapolation models, only longitudinal magnetograms have been used, neglecting a large fraction of the information about the real geometry of the surface magnetic field, and thus underestimating the total energy content of the magnetic field. Secondly, and of highest relevance for our ability to predict flares, we see less than half of the total surface of the Sun, since all of

\footnotetext{
*Corresponding author:staub@mps.mpg. de
}

our current magnetographs are looking along the Sun-Earth line.

The potential of stereoscopic viewing, which means observing the Sun from two different vantage points, has been demonstrated by the STEREO mission, but it is also recognized that the lack of magnetographic capabilities was a serious shortcoming of this mission.

Here we present the design of an instrument which aims to overcome both of these major limitations at once, the Photospheric Magnetic field Imager (PMI), a vector magnetograph to be positioned at the Lagrange point L5. From this vantage point we can extend the spatial coverage of the solar surface magnetic field to roughly $2 / 3$ of the total surface. Magnetic structures can be detected 4-5 days before they become visible from Earth, thus allowing for greatly enhanced forecast possibilities.

The aims and requirements of such an instrument have been identified during an ESA led study of the Lagrange mission. Lagrange is being developed and implemented within the framework of ESA's Space Safety Programme (Kraft et al., 2017). In order to provide a holistic set of solar monitoring data for operational space weather applications and services, the space segment carries a suite of four remote sensing instruments (coronograph, heliospheric imager, EUV imager and magnetograph), as well as four in-situ instruments (X-ray flux monitor, magnetometer, plasma analyser and radiation monitor). Being devoted to space weather monitoring, the mission puts particular 
emphasis on high reliability and availably of its near real-time data products. At the end of 2019 the instruments successfully completed their system requirements review (SRR) and got the green light for the first implementation steps by the Minesterial Council. are:

The key performance characteristics of the magnetograph

1. PMI aims to image the full solar disk with a sampling of $1 \mathrm{arcsec} / \mathrm{pixel}$, thus allowing for 2 arcsec resolution. Using a $2048 \times 2048$ pixels detector this gives sufficient margin in the field-of-view in order to cope with pointing errors of the instrument, caused by possibly imperfect spacecraft attitude control.

2. PMI is designed to deliver full magnetic field vector maps with a cadence of $30 \mathrm{~min}$. This is achieved by measuring the full polarization state of the light (full Stokes vector polarimetry) and not only the circular polarization. The vector capabilities are considered fundamental in order to improve the quality and robustness of magnetic field extrapolations (see below).

3. The dynamic range of the magnetic field strength measurements is expected to be $\pm 4 \mathrm{kG}$, in order to securely measure the fields in sunspots. A sensitivity of $10 \mathrm{G}$ is envisaged, enough to reliably detect quiet-Sun fields.

4. Additionally PMI supports the generation of high cadence data products, such as e.g. line-of-sight velocities.

An instrument with the above characteristics is not only well suited as a magnetograph for a space weather monitoring and forecasting mission like Lagrange. On top of that it possesses a large potential for solar science. Thus, it will allow reaching a better understanding and hence predictive power of solar eruptive phenomena driving space weather. In addition, with PMI for the first time novel techniques like stereoscopic magnetography and stereoscopic helioseismology can be exploited consistently on a large scale, which will open up new scientific frontiers while at the same time helping improve space weather predictions.

\section{Scientific background}

\subsection{Importance of vector magnetic maps}

Space weather events like flares and coronal mass ejections have their origin in solar active regions. Detailed knowledge of the magnetic field and electric current structure in the solar atmosphere is essential to predict the probability and strength of such eruptive events. This information can be obtained by extrapolating the photospheric magnetic field upward. For this aim vector magnetograms are essential, both on global scales and in active regions. Without further observations (synoptic) line-of-sight magnetograms allow only the computation of relative simple potential field source surface models (Schatten et al., 1969). An example is shown in Figure 1. These simple models are in a minimum energy state and do not provide any information of the free magnetic energy, which is a key quantity to estimate the amount of energy released during flares. Such estimations can be done only by taking non-potential effects into account. Recently Yeates et al. (2018) compared seven

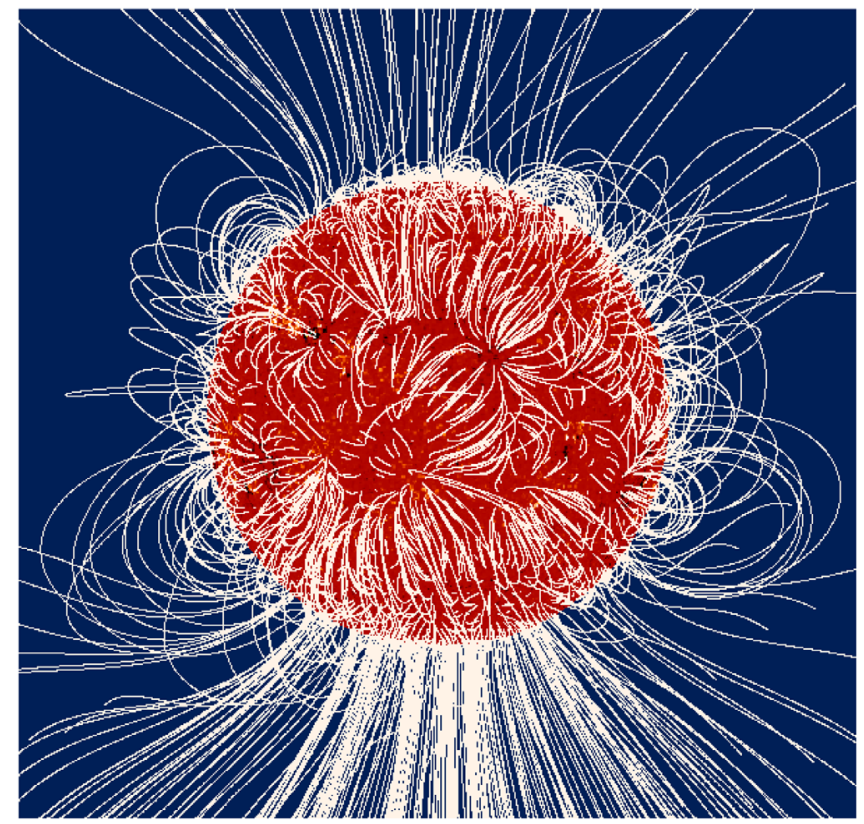

Fig. 1. A selection of magnetic field lines in a potential field source surface model computed from a synoptic magnetogram observed with SDO/HMI in March 2015. The solar surface is indicated by the red sphere.

sophisticated global non-potential corona models and found that models using vector magnetograms as boundary conditions (3 of the 7 models did that with synoptic vector maps observed with SDO/HMI) provided far more accurate reconstructions of the structure of active region magnetic fields. Several studies have used vector magnetograms for computing nonlinear force-free models of active regions in association with flares and coronal mass ejections (see, e.g. Schrijver et al., 2008; Thalmann \& Wiegelmann, 2008; Jing et al., 2009, 2010; Sun et al., 2012). A review is given by, e.g., Wiegelmann et al. (2014). These studies have been naturally limited to space weather events originating from active regions on the visible surface of the Sun. Current global models use synoptic vector magnetograms (see c.f. Tadesse et al., 2014). Such maps are created during a full solar rotation from Earth or Earth-orbiting spacecraft by combining time sequences of full disk magnetograms. This basically excludes a meaningful prediction of space weather events from the farside of the Sun. Synoptic vector maps deduced from instantaneous vector field measurements (or at least from measurements made in a shorter time than a full solar rotation) would allow incorporating all active regions of the entire solar sphere.

\subsection{Advantages of magnetography from L5}

The benefits and advantages of a space weather observing platform at the Lagrange point L5 have been recognized early and are extensively described in Vourlidas (2015). Recently, Pevtsov et al. (2020) demonstrated the usefulness of magnetogram data taken from L5 (and other Lagrange points) for the prediction quality of the quasi-steady solar wind. Operating PMI at the Lagrange L5 point promises substantial gains in our knowledge and understanding of solar magnetic fields, in 
particular in understanding the causes of and improving predictions of space weather:

1. By dint of its position following the Earth's orbit by $60^{\circ}$, a vector magnetograph at L5 will see solar magnetic activity develop already 4.5 days before it would be visible from Earth. For example, detecting and following the build-up of electric currents from L5 greatly extends the advanced warnings of pending solar eruptions and the geomagnetic storms they produce. Because the measurement of the magnetic vector is a lot more reliable near solar disk center, a vector magnetograph at L5 will be able to accurately determine the field at the footpoints of pre-eruption coronal loops that are relatively close to the eastern solar limb as seen from Earth.

2. One of the main problems besetting magnetic field measurements with the Zeeman effect is the $180^{\circ}$ ambiguity in the direction of the transverse magnetic component. Although techniques have been developed to overcome this strong shortcoming, they generally make significant assumptions regarding the structure or energetics of the magnetic field or the strength of electric currents, which may not be fully applicable exactly in the places which are most interesting from a space weather point of view. For example, the assumption that the current is minimal in the correct magnetic configuration may not necessarily be valid in active regions prior to CMEs or flares. By combining measurements of the same solar feature from two different directions (e.g. from Earth and L5) it becomes possible to remove this ambiguity without major assumptions. Since the full magnetic vector needs to be well measured to determine the energy loading of the magnetic field, removing this ambiguity is mandatory for unambiguously determining the energy that can be catastrophically released in flares or coronal mass ejections. Combining full-disk magnetic field measurements from L5 with Earth-based ones will remove the $180^{\circ}$ ambiguity for most of the eastern half of the solar disk (as seen from Earth). This and the calibration of other disambiguation techniques using such data (if necessary, for each active region individually) will greatly enhance the predictability of solar energetic events and hence the predictability of space weather events.

3. Being able to observe from two directions also has the advantage of decreasing the uncertainty in the measured magnetic vector beyond just the resolution of the $180^{\circ}$ ambiguity. Because the line-of-sight component can be determined more precisely, two of three components of the magnetic vector can be determined with small errors, instead of just one component.

4. By shortening the production time of synoptic charts, PMI at L5 together with an instrument on Earth or in Earth-orbit will allow an improved extrapolation of global magnetic fields into the corona and in particular into the heliosphere. This is needed to better predict the trajectories and travel times of coronal mass ejections. These currently suffer from the strong evolution of the magnetic field during the 27 days (a solar rotation period as seen from Earth) needed for a synoptic chart to be built up. If swathes of $\pm 30^{\circ}$ in longitude are used, then a synoptic chart can be completed in 2/3 the time needed from Earth alone, giving the Sun's magnetic field less time to evolve in the interim and hence leading to more realistic extrapolations of the magnetic field into the corona and the heliosphere.

\subsection{Role of helicity for improved flare forecasting}

The magnetic helicity is a promising quantity for the prediction of the eruptivity of active regions (ARs). This is rooted in the conservation of this quantity in ideal (resistivity being negligible) conditions, but it is also nearly conserved when non-ideal effects are significant (see, e.g., Pariat et al., 2017). Observational evidence supportive of this conclusion has been provided by (e.g. Tziotziou et al., 2012). Recently it has also been proposed that the magnetic helicity fluxes would be good predictors of solar magnetic activity over several years (Hawkes $\&$ Berger, 2018). A newly developed method, based on the conversion from the linear polarization signals in Stokes $Q$ and $U$ to the $E$ and $B$ polarization (Prabhu et al., 2020), allows the computation of a proxy for the magnetic helicity. The computation of this proxy is simple and can be implemented in the onboard data processing pipeline, and be sent to Earth with low latency and low bandwidth. This data product can be used to issue early warnings for AR eruptions, and to trigger a higher bandwidth data transmission already prior to the eruption for PMI and other solar observatories. The accurate computation of the magnetic helicity, however, requires the knowledge of the full magnetic field vector where the $180^{\circ}$ ambiguity has been resolved (see Sect. 2.2). The reliability of this resolution will benefit significantly from PMI stationed in the L5 point.

\subsection{Farside imaging}

In the absence of a solar mission at the Lagrange L3 point behind the Sun, techniques such as helioseismic holography (Lindsey \& Braun, 2000; Liewer et al., 2014) and time-distance helioseismology (Duvall et al., 1993; Zhao et al., 2019), are currently the only way to image active regions on the farside of the Sun (the hemisphere facing away from Earth at a given time), which is needed to increase forewarning of possible space weather effects beyond roughly a week. Helioseismic holography aims at imaging the Sun's three dimensional structure by numerically focusing acoustic waves at target locations in the solar interior. The traditional framework for helioseismic holography was developed in the 1990s (Lindsey \& Braun, 1990, 1997; Braun et al., 1992). Besides its impressive application to imaging the farside of the Sun, it has been used to study solar convection, active region emergence, the subsurface structure of sunspots, sources of wave excitation and sunquakes (waves caused by solar flares).

Gizon et al. (2018) and Yang (2018) report on recent efforts to develop a good theoretical basis for helioseismic holography, which have led to improvements of the method in terms of spatial resolution and signal-to-noise ratio. These developments have considerably improved our ability to detect and monitor active regions on the farside, so that active regions of average sizes can now be imaged with only a few days of HMI observations. 


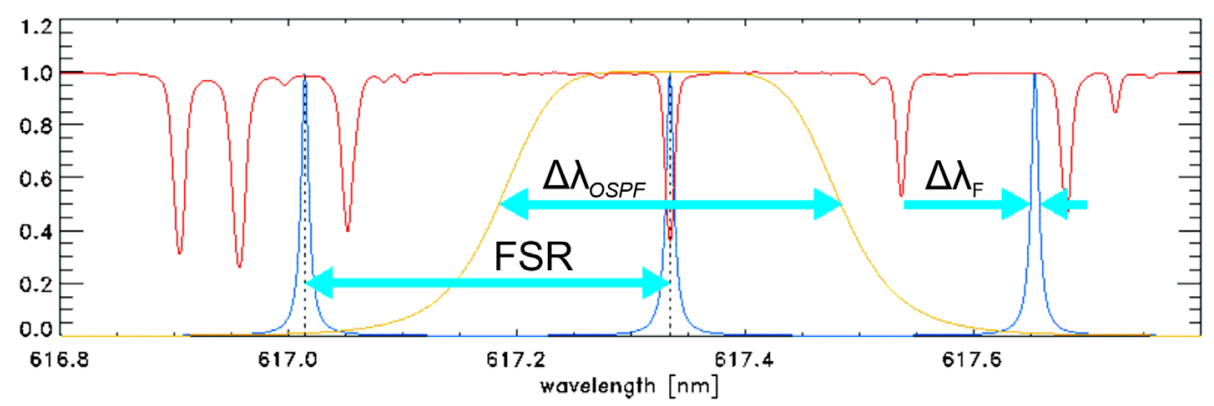

Fig. 2. Spectral filters used by PMI. Plotted are the solar spectrum around $617 \mathrm{~nm}$ (red; Fourier Transform Spectrometer (FTS) atlas; see Neckel \& Labs, 1984), tunable filter profile (blue) and bandpass of the order-sorting prefilter (OSPF) (yellow). FSR denotes the free spectral range of the tunable filter; $\Delta \lambda_{F}$ and $\Delta \lambda_{\mathrm{OSPF}}$ denote the full width at half maximum of the tunable filter and the OSPF, respectively.

Observations by PMI from L5 will greatly improve farside imaging. The holographic imaging of the areas near the limb, as seen from the Earth, currently have a reduced S/N. On the side visible by PMI the limb can be directly imaged, while on the other limb the holography performed using PMI data will be greatly improved. This improved detection of active regions will greatly enhance space weather predictions.

In addition, magnetic field measurements from L5 will help calibrate and validate the measurements of the farside made using helioseismic holography based on data taken from L1 or the Earth.

\section{Measurement principle}

PMI measures the magnetic field at the solar surface making use of the Zeeman effect, which leads to a field-strength dependent splitting of a spectral line, while the field direction marks itself in a distinctive, wavelength-dependent polarization pattern.

The measurement principle of PMI is to scan the Fe I $617.3 \mathrm{~nm}$ spectral line with a tunable narrow-band filtergraph, thus obtaining the spectral line profile at each point of the field of view, see Figure 2, (Solanki et al., 2020). The selected line is highly sensitive to the Zeeman effect (Landé factor $g=2.5$ ) and shows a clean local continuum. The same spectral line was chosen for HMI on SDO (Schou et al., 2012) and the Polarimetric and Helioseismic Imager on Solar Orbiter (Solanki et al., 2020; SO/PHI).

At each spectral scan position, the full polarization state of the incoming solar light is measured by modulating it using variable retarders. This and the subsequent polarimetric analysis encode the polarization information into intensity fluctuations.

PMI acquires a sequence of 24 images (four polarization modulation states at six wavelength positions, see Fig. 3). From these, the primary observables are derived through a series of processing steps containing classical image corrections, such as flat-field and dark correction, but also the polarimetric demodulation of the data. The result is a set of four polarized filtergrams, i.e. images of the four Stokes parameters $S=(I, Q, U, V)^{T}$ for each wavelength position.

To obtain data on physical parameters, and thus the higher level data products of PMI, a sophisticated inversion technique is applied to the observables. By inverting the radiative transfer equation (RTE, del Toro Iniesta \& Ruiz Cobo, 2016) in a fully

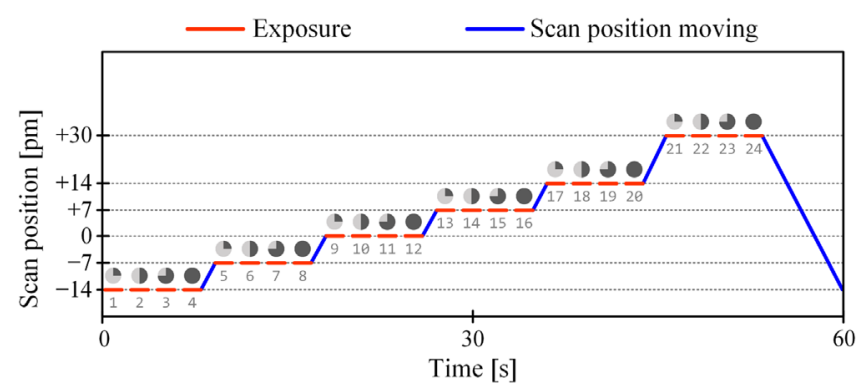

Fig. 3. Acquisition sequence followed by PMI. Numbers below red dashes correspond to image number in the sequence. Quarter circle, half circle, three quarter circle and full circle symbols correspond to four defined modulations of the polarisation state. Scan position is given with respect to the line center.

automated manner a number of physical quantities can be derived from the observables. For the particular technique proposed to be implemented in PMI, five physical quantities will be deduced: the plasma line-of-sight velocity $\left(v_{\mathrm{LOS}}\right)$, the three components of the vector magnetic field (strength, $B$, inclination relative to the line of sight, $\gamma$, and azimuth, $\phi$ ) and the continuum intensity $\left(I_{c}\right)$. The automated on-board data analysis along with other compression steps reduce the amount of data to be transferred by two orders of magnitude, allowing not only to mitigate the effects of the limitations in the telemetry of deep space missions, but also to optimise the temporal cadence of the images transmitted to Earth.

\section{Data products}

The sophisticated on-board data processing pipeline summarized in Section 3 enables several data products, which can be derived in parallel.

The primary data product of PMI (see Fig. 4) comprises the plasma line-of-sight velocity $\left(v_{\mathrm{LOS}}\right)$, the three components of the vector magnetic field (strength, $B$, inclination relative to the line of sight $(\gamma)$ and azimuth $(\phi))$ and the continuum intensity $\left(I_{c}\right)$. To comply with the allocated telemetry rate of $54 \mathrm{kbps}$, the baseline foresees to record this data product with a cadence of $30 \mathrm{~min}$. The expected latency (due to onboard processing) for delivering the data product to the spacecraft is $20 \mathrm{~min}$. 


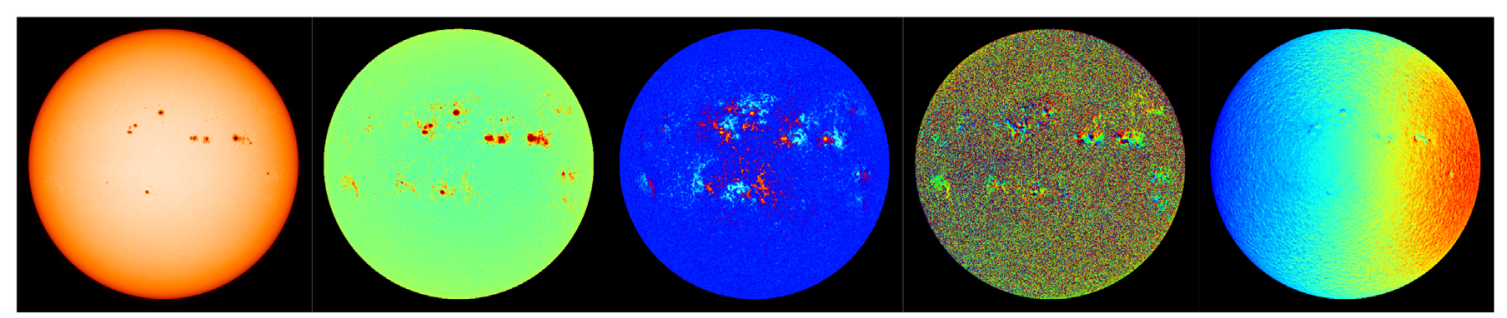

Fig. 4. Final data products (from left to right: continuum intensity, $I_{c}$; magnetic field strength, $B$; magnetic field inclination, $\gamma$; magnetic field azimuth, $\phi$ and line-of-sight velocity, $\left.v_{\mathrm{LOS}}\right)$ after on-board data analysis.

Additional (secondary) data products of PMI are raw images (offering scientific flexibility and robustness at the cost of high telemetry needs), high cadence line-of-sight velocities and longitudinal magnetic field maps computed on board (either by inversions or using simplified algorithms). Given that only very limited onboard processing resources are required for these additional data products, potential cadences are mainly limited by the available telemetry.

\section{Technical implementation}

PMI is a diffraction limited, wavelength tunable, quasimonochromatic, polarization sensitive imager that will provide full-disk images of the Sun and achieve an angular resolution of about 2 arcsec, with a pixel size of 1 arcsec.

\subsection{Functional layout}

PMI consists of two units: the optics unit and the electronics unit. The optics unit includes as main subsystems the optical telescope assembly, the polarization modulation package (PMP), the filtergraph system, and the focal plane assembly (FPA). The electronics unit is formed by four modules: the power converter module (PCM), the digital processing unit (DPU), the analog motor and heaters drivers board (AMHD), and the tip-tilt controller board (TTC). Figure 5 shows an overview of the different functional groups and subsystems in PMI.

\subsection{Optics unit}

The design of the PMI optics unit is derived from the SO/PHI development, see Solanki et al. (2020). The telescope assembly is in many ways a scaled version of the SO/PHI high resolution channel (HRT, Gandorfer et al., 2018), which has allowed the shrinking of the unit with respect to SO/PHI, both in terms of volume $\left(500 \times 400 \times 700 \mathrm{~mm}^{3}\right.$ envelope, closed aperture door) and mass $(22.6 \mathrm{~kg}$ without margin, taking into account additional shielding due to the external accommodation of the unit, the placement of the entrance filter directly on the unit instead of on the spacecraft's heatshield as in $\mathrm{SO} / \mathrm{PHI}$, etc.).

To account for its external accommodation, the optics unit has an aperture door mechanism. The door is aimed mainly to protect the optics unit against contaminants during ground activities, and during launch and early orbit phase operations. The door is moved by a multi-use mechanism with a fail-safe functionality. In case of malfunctioning, a pin puller is released and the door is opened to its maximum actuation range of $180^{\circ}$.

The primary structure of the PMI optics unit has as core elements two structural blocks and six carbon fiber reinforced plastic (CFRP) struts between them. The blocks are made of milled AlBeMet ${ }^{\circledR}$ AM162, a high stiffness to mass ratio aluminum-beryllium metal matrix composite, with higher thermal conductivity and lower coefficient of thermal expansion than typical spaceborne aluminum alloys. The design of the primary structure ensures the required alignment stability of the telescope elements.

The cavity between the two structural blocks is closed laterally by four CFRP sandwich panels. These panels allow the contamination control of the optical elements inside and support some proximity electronics. Since the unit is externally mounted to the spacecraft, it needs to be thermally insulated from the cold space. This is achieved by multi-layer insulation blankets that wrap the unit; these blankets use the sandwich panels as support structure. The PMI optics unit primary structure, sandwich panels and subsystems are depicted in Figure 6.

The first element in the instrument's optical path is the entrance window, which is located at the entrance pupil and mounted on the structural front block. The PMI window is based on the SO/PHI HRT entrance window. It consists of two Suprasil ${ }^{\circledR}$ glasses, mounted in a titanium flange. The glasses carry in total four different multilayer coatings. The combination of the four coatings forms a band-pass filter, allowing only the transmission of a band of $30 \mathrm{~nm}$ around the science wavelength, meaning that less than $4 \%$ of the solar power reaching the entrance window actually enters the PMI optics unit.

The telescope assembly is designed as a two-mirror off-axis Ritchey-Chrétien telescope and provides a field of view of 34.5 arcmin (Table 1), achieving an angular resolution of approximately 2 arcsec. It has an entrance pupil diameter of $75 \mathrm{~mm}$ and is composed of two aspheric Zerodur ${ }^{\circledR}$ mirrors. The secondary mirror is mounted on a dynamic tip-tilt actuator acting as a compensator for image jitter and is thus part of the instrument's image stabilization system (ISS).

The tip-tilt mirror is complemented by a limb sensor, whose light path is coupled out from the main beam after the telescope assembly. It therefore shares the most alignment sensitive part of the instrument with the science beam and generates a correction signal for the tip-tilt mechanism closing the control loop of the ISS. To achieve its high quality data products (Sect. 4), PMI performs differential photometry making it particularly vulnerable to image jitter. To keep the cross-talk below the required polarimetric precision of $10^{-3}$, the maximal tolerable shift between images has to be kept below 0.05 arcsec. The ISS 


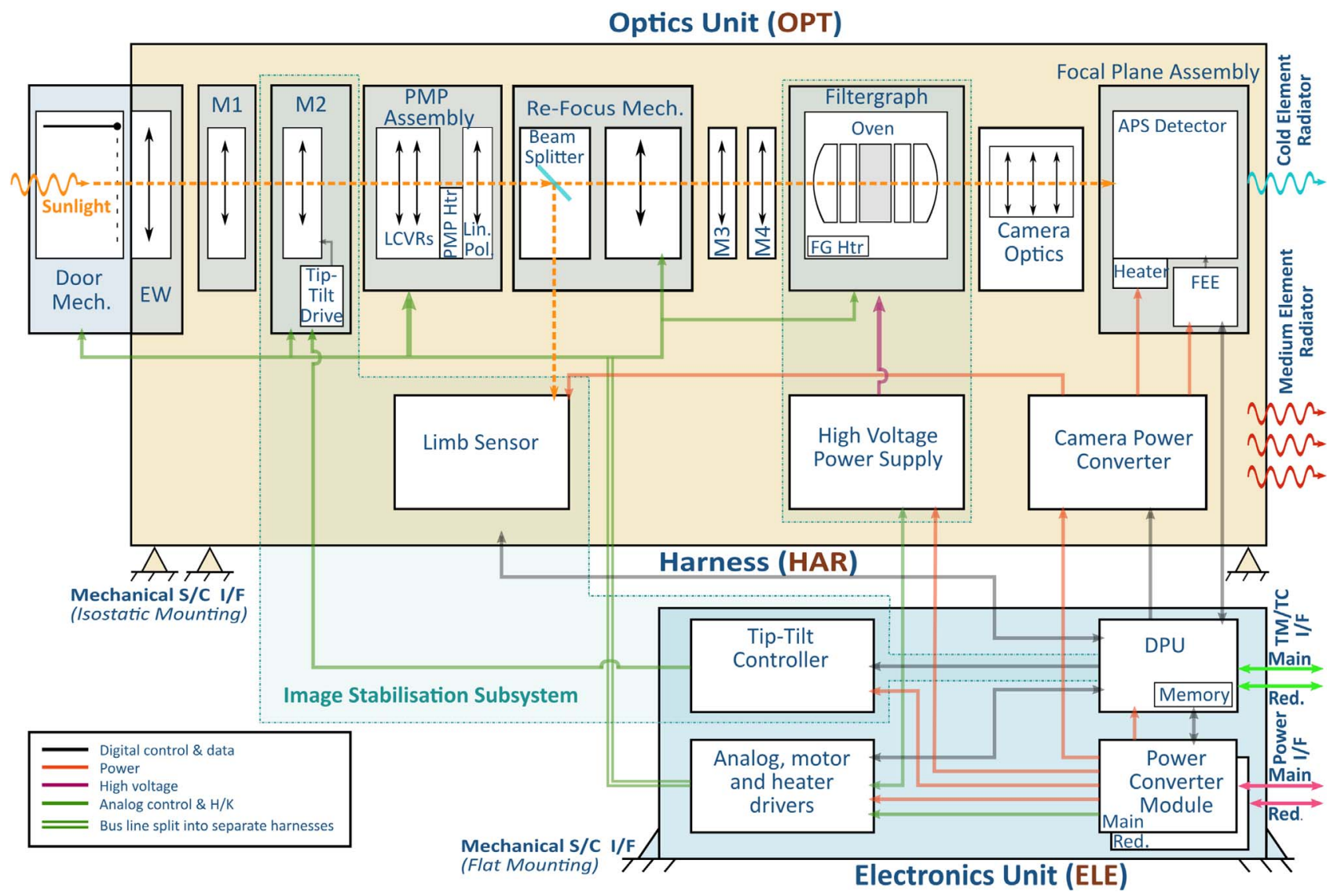

Fig. 5. Schematic overview of the PMI units and functional blocks (EW: entrance window, PMP: polarization modulation package, FEE: front end electronics).

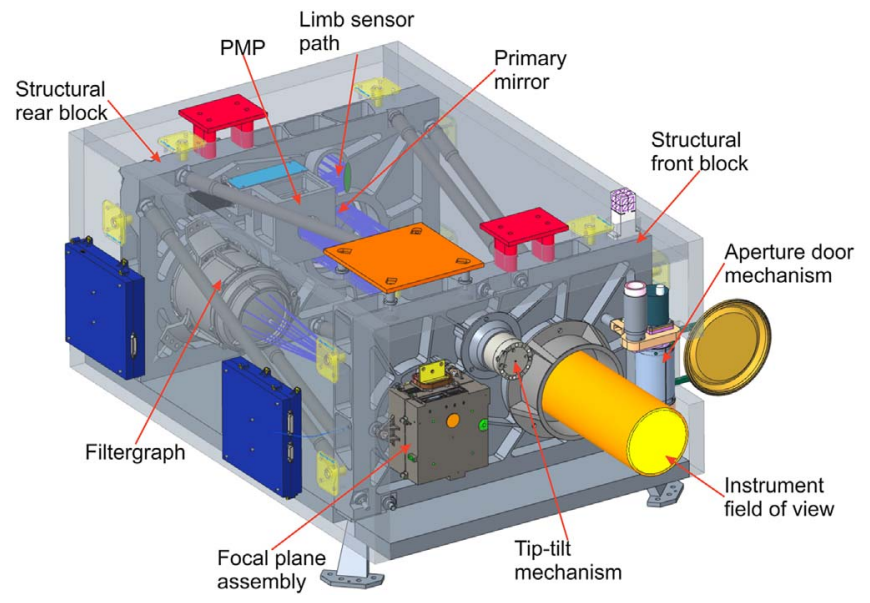

Fig. 6. Preliminary configuration of the optics unit.

therefore plays a crucial role in ensuring the instrument performance, while keeping the requirements on the spacecraft moderate.

The next key subsystem along the optical path is the polarization modulation package, which performs the polarization analysis of the incoming light, see Alvarez-Herrero et al. (2017). In order to achieve the four different polarization modulations, required to infer the full Stokes vector of the incoming
Table 1. Optical parameters.

\begin{tabular}{lcc}
\hline Parameter & Symbol & Value \\
\hline Working wavelength & $\lambda_{0}$ & $617.3 \mathrm{~nm}$ \\
Effective focal length & $f_{\text {eff }}$ & $1844.6 \mathrm{~mm}$ \\
Field of view & $\alpha_{\mathrm{FOV}}$ & $\emptyset 0.57^{\circ}$ \\
Entrance pupil $\varnothing$ & $D$ & $75 \mathrm{~mm}$ \\
Detector size & Pixels & $2048 \times 2048$ \\
Plate scale & $\alpha_{\text {pixel }}$ & $1^{\prime \prime} 00$ \\
Image quality & $\sigma_{\mathrm{WFE}}$ & $\leq \lambda_{0} / 14$ \\
\hline
\end{tabular}

light, the PMP consists of two nematic liquid crystal variable retarders. These are followed by a linear polarizer (the analyzer), which translates the polarization signal into intensity changes, detectable by the instrument's focal plane assembly. As part of the on-board processing the so obtained coupled polarization states are demodulated by solving a set of linear equations to obtain the Stokes vector of each pixel (for more information on polarization modulation and demodulation techniques, we refer to del Toro Iniesta, 2003). The retardance introduced by the liquid crystals is temperature dependent. Thus to ensure the high polarimetric efficiencies, the PMP has an optimized thermo-mechanical design with dedicated sensor controlled heaters for each of the two liquid crystals, achieving a thermal stability of $\pm 0.5^{\circ} \mathrm{C}$. 
Before reaching the science detector, the light goes through the re-focus mechanism and the Fabry-Perot interferometer. The re-focus mechanism is based on the SO/PHI heritage. It compensates for focus shifts due to manufacturing and alignment errors, and thermo-mechanical distortions in flight. The latter effect is expected to be much smaller than in SO/PHI, since the distance to the Sun once in nominal position will be nearly constant at L5. A magnifier lens is used as de-focus compensator. It can be moved over a range of $\pm 10 \mathrm{~mm}$ along the optical path with a positioning accuracy of $<0.1 \mathrm{~mm}$ through a stepper motor driven drivetrain relying on a miniature ballscrew and a worm gear stage. The lens is mounted into a translation stage, which is guided by high precision linear bearings in order to prevent misalignments of the lens while focusing. The focus is controlled using an autonomous on-board re-focusing procedure.

The filtergraph is the heart of the instrument. It accommodates the Fabry-Perot interferometer in a stabilized thermal environment $(30 \mathrm{mK}$ around the design temperature of the etalon). The Fabry-Perot interferometer is based on a solid state etalon $\left(\mathrm{LiNbO}_{3}\right)$ used in telecentric configuration and two order sorting prefilters. The etalon can be tuned through the application of high voltage in order to sample the $\mathrm{Fe}$ I $617.3 \mathrm{~nm}$ spectral line (Fig. 2).

The wavelength resolution of PMI is optimized for the standard observing mode (dynamic range: $\pm 4 \mathrm{kG}$ ) and an analysis method based on Milne-Eddington type inversions. To achieve critical sampling of the spectral line in five wavelength positions $\pm 14 \mathrm{pm}$ around the line core position plus the continuum at $+30 \mathrm{pm}$ (Fig. 3), the narrow-band images produced by the etalon have therefore a full-width-half-maximum transmission in the range of 10-14 pm.

The last subsystem and final destination of the light path is the Focal Plane Assembly (FPA). The heart of the the FPA is a $2048 \times 2048$ pixels active pixel sensor (APS) detector. The exposure time is set by the electronic shutter of the detector that ensures repeatability for all the images with an accuracy of better than $10 \mathrm{ppm}$ (needed for accurate differential polarimetry).

\subsection{Electronics unit}

As for the optics unit, the PMI electronics unit inherits many characteristics from the SO/PHI electronics unit. The PMI electronics unit is internally mounted in the spacecraft, and follows a modular design concept, as shown in Figure 7. It comprises the main and redundant power converter modules, digital processing unit, tip-tilt mechanism controller and the analog mechanisms and heaters driver. Compared to SO/PHI, the stacked structural concept profits from the overall configuration optimization and shows (slightly) reduced values for mass $\left(5.6 \mathrm{~kg}\right.$, no margin) and envelope $\left(225 \times 232 \times 175 \mathrm{~mm}^{3}\right)$.

The PCM receives the power from the spacecraft through a regulated bus at approximately $28 \mathrm{~V}$, and distributes it to the rest of the instrument, at the required voltages. The maximum instrument power consumption is estimated at $40 \mathrm{~W}$. Whereas TTC, PCM and AMHD follow closely the SO/PHI heritage design, the DPU design needs to reflect the increased requirements of an operational space weather mission compared to a scientific mission (i.e. reliability, latency, etc.).

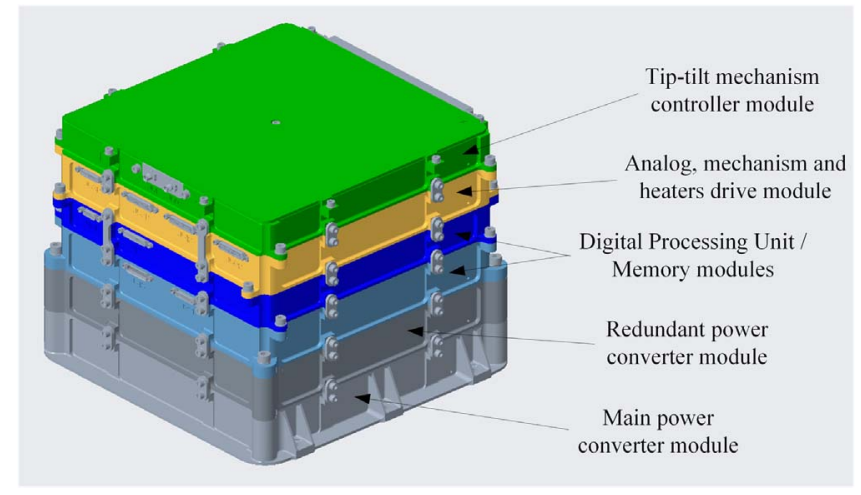

Fig. 7. Preliminary configuration of the electronics unit.

The SO/PHI DPU was designed to process most of the acquired scientific data outside the observation windows, so no real time processing was required. Unlike SO/PHI, PMI is required to post-process the data right after the acquisition, as mentioned in Section 4. The required cadence and latency of the primary data products impose a huge computational burden on the DPU, which needs to acquire and accumulate the images, perform classical post-processing operations (flat field and dark field correction, for instance) and the polarization demodulation, run the RTE inversion and compress the resulting magnetograms in less than the 20 min latency.

To answer the need for near real time processing and demanding reliability figures, the DPU architecture is based on state of the art re-configurable field-programmable gate arrays for the on-board processing pipeline, enabling (partial) triple-mode redundancy implementation of the code. It further consists of a system controller for instrument control and communications, and a combination of several types and sizes of memory for storing and buffering science data products, calibration data and instrument software. Communication with the spacecraft is made through a redundant SpaceWire connection.

\subsection{Instrument performance aspects}

Achieving the required magnetic field sensitivity of $10 \mathrm{G}$ requires mainly a high repeatability of the measurement. This is achieved by a high shutter repeatability to ensure constant exposure times $(\leq 10 \mathrm{ppm})$, a well calibrated instrumental polarization and low polarization modulation errors. A simulation performed for the SO/PHI instrument by Martinez Pillet (2007), involving random, small amounts of instrumental polarization (such as from entrance windows, few degrees off-axis mirrors, etc.), a $10 \mathrm{ppm}$ shutter repeatability, and phase errors of $1^{\circ}$ in the actual retardances of the liquid-crystal devices, confirms that the accuracy for the longitudinal magnetic field is $0.1 \mathrm{G}$. The same simulation provides an accuracy for the transverse magnetic field of $14 \mathrm{G}$.

Realistic simulations of the instrument performance, using numerical experiments and theoretical calculations based on response functions (Orozco Suárez \& Del Toro Iniesta, 2007), demonstrate that the minimum values detectable are $3.7 \mathrm{G}$ (longitudinal), $52 \mathrm{G}$ (transversal), and $7 \mathrm{~ms}^{-1}$ (line-ofsight velocity) for a photon noise level of $10^{-3}$. With a high 
repeatability of the individual measurements a temporal averaging allows to reduce this noise level and therefore decrease the minimum detectable values further.

\section{Outlook and conclusion}

The PMI instrument builds closely on the heritage accumulated from the recently successfully launched SO/PHI instrument on board Solar Orbiter. It therefore profits from several critical technology developments, which have now demonstrated their in-orbit capabilities. Compared to its heritage it is adapted to the specifics of an operational space weather mission, such as low latency, reliability, etc., while further optimizing its resource demands (i.e. mass, volume).

PMI is capable of delivering maps of the full magnetic field vector at an angular resolution of 2 arcsec with a cadence of $30 \mathrm{~min}$, while coping with the expected telemetry limitations from the Lagrange point L5. It thus answers a central demand of the space weather community and contributes to improved space weather forecasting capabilities.

In addition to its value for operational space weather data products, having PMI observations from L5 in combination with observations from Earth (such as those gathered by HMI on SDO) opens up new opportunities for scientific investigations.

Acknowledgements. The PMI team would like to thank the ESA and RAL Lagrange teams for their cooperative support. The work on PMI was funded through ESA as part of the Lagrange Missions Remote Sensing Instruments Phase A/B1 study and by the President of the Max Planck Society (MPG). This work has been partially supported by the BK21 plus program through the National Research Foundation (NRF) funded by the Ministry of Education of Korea. The editor thanks Philip Scherrer and an anonymous reviewer for their assistance in evaluating this paper.

\section{References}

Alvarez-Herrero A, Parejo PG, Laguna H, Villanueva J, Barandiarán J, Bastide L, Reina M, Royo M. 2017. The polarization modulators based on liquid crystal variable retarders for the PHI and METIS instruments for the solar orbiter mission. In: International Conference on Space Optics - ICSO 2014, Sodnik Z, Cugny B, Karafolas $\mathrm{N}$ (Eds.), International Society for Optics and Photonics, SPIE, Vol. 10563, pp. 850-858. https://doi.org/10.1117/12.2304224.

Braun DC, Lindsey C, Fan Y, Jefferies SM. 1992. Local acoustic diagnostics of the solar interior. Astrophys $J$ 392: 739-745. https://doi.org/10.1086/171477.

del Toro Iniesta JC. 2003. Introduction to spectropolarimetry, Cambridge University Press, Cambridge, UK. https://doi.org/ 10.1017/CBO9780511536250.

del Toro Iniesta JC, Ruiz Cobo B. 2016. Inversion of the radiative transfer equation for polarized light. Living Rev Sol Phys 13(1): 4. https://doi.org/10.1007/s41116-016-0005-2.

Duvall TL Jr, Jefferies SM, Harvey JW, Pomerantz MA. 1993. Timedistance helioseismology. Nature 362(6419): 430-432. https://doi. org/10.1038/362430a0.
Gandorfer A, Grauf B, Staub J, Bischoff J, Woch J, et al. 2018. The High Resolution Telescope (HRT) of the Polarimetric and Helioseismic Imager (PHI) Onboard Solar Orbiter. In: Space telescopes and instrumentation 2018: Optical, infrared, and millimeter wave, Vol. 10698 of Proc. SPIE, 106984N. https://doi.org/0.1117/12.2311816.

Gizon L, Fournier D, Yang D, Birch AC, Barucq H. 2018. Signal and noise in helioseismic holography. A\&A 620: A136. https://doi.org/ 10.1051/0004-6361/201833825.

Hawkes G, Berger MA. 2018. Magnetic helicity as a predictor of the solar cycle. Sol Phys 293(7): 109. https://doi.org/10.1007/s11207018-1332-3.

Jing J, Chen PF, Wiegelmann T, Xu Y, Park S-H, Wang H. 2009. Temporal evolution of free magnetic energy associated with four X-class flares. Astrophys J 696(1): 84-90. https://doi.org/10.1088/ 0004-637X/696/1/84.

Jing J, Tan C, Yuan Y, Wang B, Wiegelmann T, Xu Y, Wang H. 2010. Free magnetic energy and flare productivity of active regions. Astrophys J 713(1): 440-449. https://doi.org/10.1088/ 0004-637X/713/1/440.

Kraft S, Puschmann KG, Luntama JP. 2017. Remote sensing optical instrumentation for enhanced space weather monitoring from the L1 and L5 Lagrange points. In: Society of Photo-Optical Instrumentation Engineers (SPIE) Conference Series, Vol. 10562 of Society of Photo-Optical Instrumentation Engineers (SPIE) Conference Series, 105620F. https://doi.org/10.1117/12.2296100.

Lagg A, Lites B, Harvey J, Gosain S, Centeno R. 2017. Measurements of photospheric and chromospheric magnetic fields. Space Sci Rev 210(1-4): 37-76. https://doi.org/10.1007/s11214-0150219-y.

Liewer PC, González Hernández I, Hall JR, Lindsey C, Lin X. 2014. Testing the reliability of predictions of far-side active regions from helioseismology using STEREO far-side observations of solar activity. Sol Phys 289: 3617-3640. https://doi.org/10.1007/ s11207-014-0542-6.

Lindsey C, Braun DC. 1990. Helioseismic imaging of sunspots at their antipodes. Sol Phys 126: 101-115. https://doi.org/10.1007/ BF00158301.

Lindsey C, Braun DC. 1997. Helioseismic holography. Astrophys $J$ 485: 895-903.

Lindsey C, Braun DC. 2000. Seismic images of the far side of the Sun. Science 287: 1799-1801. https://doi.org/10.1126/science. 287.5459.1799.

Martinez Pillet V. 2007. Instrumental approaches to magnetic and velocity measurements in and out of the ecliptic plane. In: ESA Special Publication, Vol. 651 of ESA Special Publication, 27 p.

Neckel H, Labs D. 1984. The solar radiation between 3300 and 12500 A. Sol Phys 90: 205-258. https://doi.org/10.1007/ BF00173953.

Orozco Suárez D, Del Toro Iniesta JC. 2007. The usefulness of analytic response functions. $A \& A$ 462: 1137-1145. https://doi.org/ 10.1051/0004-6361:20066201.

Pariat E, Leake JE, Valori G, Linton MG, Zuccarello FP, Dalmasse K. 2017. Relative magnetic helicity as a diagnostic of solar eruptivity. A\&A 601: A125. https://doi.org/10.1051/00046361/201630043.

Pevtsov AA, Petrie G, MacNeice P, Virtanen II. 2020. Effect of additional magnetograph observations from different Lagrangian points in Sun-Earth system on predicted properties of quasi-steady solar wind at 1 AU. Space Weather 18(7): e02448. https://doi.org/ 10.1029/2020SW002448.

Prabhu A, Brandenburg A, Käpylä MJ, Lagg A. 2020. Helicity proxies from linear polarisation of solar active regions. A\&A 641: A46. https://doi.org/10.1051/0004-6361/202037614. 
Schatten KH, Wilcox JM, Ness NF. 1969. A model of interplanetary and coronal magnetic fields. Sol Phys 6(3): 442-455. https://doi. org/10.1007/BF00146478.

Schou J, Scherrer PH, Bush RI, Wachter R, Couvidat S, et al. 2012. Design and ground calibration of the helioseismic and magnetic imager (HMI) instrument on the solar dynamics observatory (SDO). Sol Phys 275(1-2): 229-259. https://doi.org/10.1007/ s11207-011-9842-2.

Schrijver CJ, DeRosa ML, Metcalf T, Barnes G, Lites B, et al. 2008. Nonlinear force-free field modeling of a solar active region around the time of a major flare and coronal mass ejection. Astrophys $J$ 675(2): 1637-1644. https://doi.org/10.1086/527413.

Solanki SK, del Toro Iniesta JC, Woch J, Gandorfer A, Hirzberger J, et al. 2020. The polarimetric and helioseismic imager on solar orbiter. A\&A 642: A11. https://doi.org/10.1051/0004-6361/ 201935325.

Sun X, Hoeksema JT, Liu Y, Wiegelmann T, Hayashi K, Chen Q, Thalmann J. 2012. Evolution of magnetic field and energy in a major eruptive active region based on SDO/HMI observation. Astrophys $J$ 748(2): 77. https://doi.org/10.1088/0004-637X/748/2/77.

Tadesse T, Wiegelmann T, Gosain S, MacNeice P, Pevtsov AA. 2014. First use of synoptic vector magnetograms for global nonlinear, force-free coronal magnetic field models. A\&A 562: A105. https://doi.org/10.1051/0004-6361/201322418.
Thalmann JK, Wiegelmann T. 2008. Evolution of the flaring active region NOAA 10540 as a sequence of nonlinear force-free field extrapolations. $A \& A$ 484(2): 495-502. https://doi.org/10.1051/ 0004-6361:200809508.

Tziotziou K, Georgoulis MK, Raouafi N-E. 2012. The magnetic energy-helicity diagram of solar active regions. Astrophys J Lett 759(1): L4. https://doi.org/10.1088/2041-8205/759/1/L4.

Vourlidas A. 2015. Mission to the Sun-Earth L5 Lagrangian point: An optimal platform for space weather research. Space Weather 13(4): 197-201. https://doi.org/10.1002/2015SW001173.

Wiegelmann T, Thalmann JK, Solanki SK. 2014. The magnetic field in the solar atmosphere. A\&A Rev. 22: 78. https://doi.org/10.1007/ s00159-014-0078-7.

Yang D. 2018. Modeling experiments in helioseismic holography. Ph.D. Thesis, Georg-August-Universität Göttingen, Göttingen, Germany http://hdl.handle.net/21.11130/00-1735-0000-0003-C115-B.

Yeates AR, Amari T, Contopoulos I, Feng X, Mackay DH, et al. 2018. Global non-potential magnetic models of the solar corona during the March 2015 eclipse. Space Sci Rev 214(5): 99. https://doi.org/10.1007/s11214-018-0534-1.

Zhao J, Hing D, Chen R, Hess Webber S. 2019. Imaging the Sun's far-side active regions by applying multiple measurement schemes on multiskip acoustic waves. Astrophys $J$ 887(2): 216. https://doi. org/10.3847/1538-4357/ab5951.

Cite this article as: Staub J, Fernandez-Rico G, Gandorfer A, Gizon L, Hirzberger J, et al. 2020. PMI: The Photospheric Magnetic Field Imager. J. Space Weather Space Clim. 10, 54. https://doi.org/10.1051/swsc/2020059. 